\title{
A macrophage-stimulating compound from a screen of microbial natural products
}

\author{
Julie A Perry ${ }^{1,2}$, Kalinka Koteva ${ }^{1,2}$, Chris P Verschoor ${ }^{1,3}$, Wenliang Wang ${ }^{1,2}$, Dawn ME Bowdish ${ }^{1,3}$ and \\ Gerry D Wright ${ }^{1,2,3}$
}

\begin{abstract}
Rising rates of antibiotic resistance in bacterial pathogens is a medical crisis of global concern that necessitates the development of new treatment strategies. We have isolated a natural product with macrophage-stimulating activity from a screen of microbially produced bioactive molecules. Streptazolin increased bacterial killing and elaboration of immunostimulatory cytokines by macrophages in vitro. Furthermore, we show that streptazolin stimulates the macrophage nuclear factor кВ (NF-кB) pathway via phosphatidylinositide 3-kinase (PI3K) signaling, and that the conjugated diene moiety is essential for stimulatory activity. Immunostimulatory molecules like streptazolin represent entries into new treatment paradigms to address the challenge of antibiotic resistance.
\end{abstract}

The Journal of Antibiotics (2015) 68, 40-46; doi:10.1038/ja.2014.83; published online 2 July 2014

\section{INTRODUCTION}

There is an ever-growing number of pathogenic bacteria resistant to multiple antibiotics. The result is a public health crisis of global impact. This fact is spurring calls not only for new antibiotic drugs but also for therapeutic alternatives that may be less prone to resistance over the long term. Noncanonical strategies such as the pursuit of combinations of antibiotics with adjuvants and inhibitors of resistance, targeting virulence and exploring immunostimulatory strategies to harness our innate capacity to overcome infection are examples.

The body's first line of defense against invading pathogens is the innate immune system that depends strongly on professional phagocytes to engulf and destroy invading pathogens. ${ }^{1}$ Among these professional immune cells are the macrophages: phagocytic and antigen-presenting cells, derived from blood monocytes (with selfproliferating capacity) and found throughout the body. ${ }^{1}$ Macrophages are equipped with a broad range of pattern-recognition receptors (which include the Toll-like receptors (TLRs)) to recognize structures associated with invading pathogens or with necrosis or apoptosis of host cells. ${ }^{2}$ TLR signaling triggers translocation of the master transcriptional regulator NF- $\kappa \mathrm{B}$ (nuclear factor $\kappa \mathrm{B}$ ) to the nucleus, where it governs the expression of proinflammatory cytokines (for example, tumor necrosis factor $\alpha$ (TNF $\alpha$ ), interleukin (IL)-1 and IL-6) and chemokines (for example, macrophage inflammatory protein-1 $\alpha){ }^{3} \quad$ Macrophage-produced cytokines and chemokines provide a crucial bridging point between the innate and the adaptive immune systems, making these cells an excellent target for immunostimulatory strategies.
We reasoned, based on our growing understanding of the response of the innate immune system to microbially produced biomolecules such as lipopolysaccharide (LPS), cationic peptides and reported precedent, ${ }^{4}$ that small molecules produced by microorganisms themselves might have such immune-enhancing properties (see Hancock et al..$^{5}$ for an excellent review of immunomodulatory therapies). To find molecules with macrophage-stimulating capabilities, we developed a screening protocol and screened a large in-house collection of extracts from Actinobacteria for molecules with the ability to activate the NF- $\kappa B$ pathway and increase macrophagemediated killing of bacteria. Here we report the identification of the small-molecule streptazolin as a novel macrophage-stimulating small molecule.

\section{RESULTS}

Developing a screening method for macrophage-activating small molecules

We developed a screen for macrophage activation using a human macrophage cell line (THP-1 Blue, Invivogen, San Diego, CA, USA) expressing a secreted alkaline phosphatase (SEAP) reporter gene under the control of the master transcriptional regulator NF- $\kappa B$. Using the SEAP reporter assay allowed us to perform a highthroughput screen of a diverse in-house collection of actinomycete culture extracts for molecules able to activate macrophages. We found that $\sim 25 \%$ of extracts screened (139/580 total) induced THP-1 Blue $\mathrm{NF}-\kappa \mathrm{B}$ reporter expression at a level equal to or higher than that induced by an equal volume of a heat-killed bacterium (Listeria monocytogenes, abbreviated henceforth as HKLM), used as positive

${ }^{1}$ M.G. DeGroote Institute for Infectious Disease Research, McMaster University, Hamilton, Ontario, Canada; ${ }^{2}$ Department of Biochemistry and Biomedical Sciences, DeGroote School of Medicine, Hamilton, Ontario, Canada and ${ }^{3}$ Department of Pathology and Molecular Medicine, McMaster University, Hamilton, Ontario, Canada

Correspondence: Professor GD Wright, M.G. DeGroote Institute for Infectious Disease Research, McMaster University, 1280 Main Street West, Hamilton, Ontario, Canada L8S 4K1.

E-mail: wrightge@mcmaster.ca

Received 20 December 2013; revised 20 May 2014; accepted 28 May 2014; published online 2 July 2014 
control. Furthermore, 19\% of extracts induced macrophage differentiation as assessed by microscopy. Less than $5 \%$ of extracts were toxic to the macrophages (as assessed by Trypan Blue staining and microscopy), and $13 \%$ had unspecified antibiotic activity against the model human commensal bacterium Streptococcus mutans. Thirtyeight extracts found to induce very high levels of NF- $\kappa \mathrm{B}$ expression were used in a follow-up study to assess the ability of extract to induce macrophage-mediated killing of $S$. mutans. Positive extracts were counter-screened for their effect on the rate of bacterial uptake by macrophage and antibiotic activity, and found to be no different than positive controls (data not shown).

\section{Purification of streptazolin, a novel macrophage-stimulating molecule}

We chose to pursue strain WAC1325 (closest 16S rRNA in GenBank is Streptomyces sp. K35-27P) for purification based on its ability to stimulate NF- $\kappa B$ to high levels and increase the rate of macrophagemediated killing of intracellular bacteria (Figure 1). Production of the macrophage-activating compound by strain WAC1325 was then optimized and found to occur in greatest abundance in liquid MGY medium following 6 days of incubation at $30^{\circ} \mathrm{C}$ with agitation. Both cell pellet and supernatant were extracted with ethyl acetate, and separated on a CombiFlash (Teledyne Isco, Lincoln, NE, USA) system using a silica resin and chloroform $/ 10 \%$ methanol in chloroform as solvent system. NF- $\kappa \mathrm{B}$ activity was assessed at all stages of purification using the SEAP reporter assay. Purified active compound was subjected to high-resolution MS/MS, 1-D and 2-D NMR experiments that confirmed the compound structure to be that of the previously reported small-molecule streptazolin (Figure 2a, Table 1; HR-MS data: streptazolin calculated for $\mathrm{C}_{11} \mathrm{H}_{14} \mathrm{NO}_{3}[\mathrm{M}+\mathrm{H}]^{+} 208.0974 \mathrm{Da}$; found 208.0977 Da). Titration experiments revealed that streptazolin stimulated the NF- $\kappa B$ pathway to levels equivalent to HKLM controls at concentrations between 60 and $130 \mu \mathrm{g} \mathrm{ml}^{-1}$, which is comparable to other natural product immunomodulatory molecules, ${ }^{6,7}$ and had no antibiotic activity at concentrations tested up to $512 \mu \mathrm{g} \mathrm{ml}^{-1}$.

\section{Structure/activity characterization of streptazolin}

Streptazolin has been documented to polymerize in concentrated form, likely because of the reactivity of the conjugated diene moiety. ${ }^{8,9}$

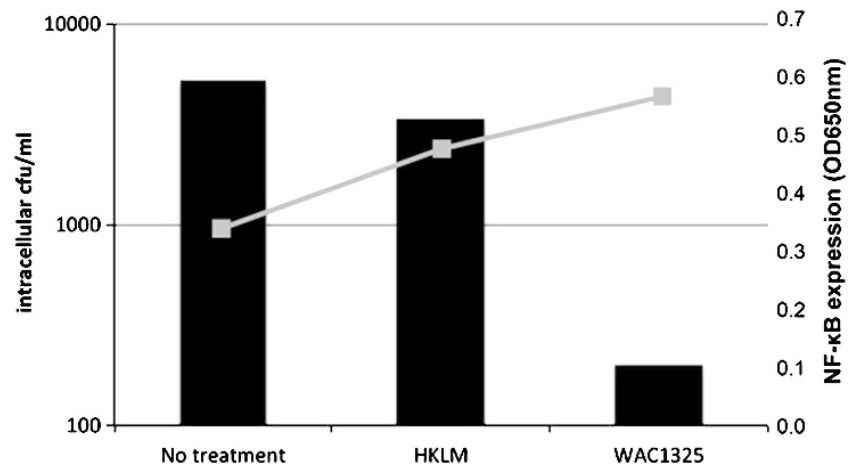

Figure 1 Strain WAC1325 was identified by high-throughput screening as producing a macrophage-activating molecule. A collection of 560 extracts from the culture supernatants of actinobacteria were screened for their ability to induce nuclear factor $\kappa \mathrm{B}(\mathrm{NF}-\kappa \mathrm{B})$ reporter activity in THP-1 Blue macrophages. Extracts inducing high-level reporter activity were further assessed for their ability to phagocytose and kill Streptococcus mutans. NF-KB activity (gray squares); corresponding intracellular bacterial CFU per $\mathrm{ml}$ (black bars) for the negative control, heat-killed Listeria monocytogenes (HKLM)-positive control and top hit strain WAC1325 are shown.
We also found the molecule to be highly unstable, and decided to investigate the structure/activity relationship of the diene. We prepared stable tetrahydrostreptazolin by reduction of the natural product using the protocol of Kozikowski and Park ${ }^{9}$ (HR-MS: tetrahydrostreptazolin calculated for $\mathrm{C}_{11} \mathrm{H}_{18} \mathrm{NO}_{3}[\mathrm{M}+\mathrm{H}]^{+}$ $212.1287 \mathrm{Da}$; found $212.1284 \mathrm{Da}$ ), and assayed it for $\mathrm{NF}-\kappa \mathrm{B}-$ stimulatory activity in parallel with active streptazolin (concentrations tested between 30 and $240 \mu \mathrm{g} \mathrm{ml}^{-1}$; streptazolin most active at a concentration of $60 \mu \mathrm{g} \mathrm{ml}^{-1}$ ), but the reduced compound showed no activity at any concentrations tested (data not shown). Based on this assay and the work of others, ${ }^{8}$ we propose that streptazolin activity is due to the intrinsic reactivity of its conjugated diene system (Figure 2).

\section{Streptazolin 'primes' macrophages for killing}

Because one of the most important functions of macrophages may be the induction of the acute inflammatory response via the elaboration of cytokines, we next assessed the cytokine-stimulating potential of purified streptazolin on THP-1 Blue cells by enzyme-linked immunosorbent assay (ELISA). Streptazolin alone does not induce TNF $\alpha$ secretion, but instead significantly boosts the TNF $\alpha$ response to LPS (Figure 3). Similarly, streptazolin significantly boosts the elaboration of IL- 8 in the presence of LPS in addition to having a stimulatory effect on IL-8 secretion on its own. Minor increases in the secretion of both cytokines were also observed when a combination of streptazolin and the synthetic TLR2 agonist Pam3CSK4 was used to treat cells. Finally, adding purified streptazolin in conjunction with HKLM resulted in higher NF- $\kappa B$ expression than streptazolin alone (Figure 4), suggesting that streptazolin may have a role in priming macrophages for response to bacterial agonists.

\section{Streptazolin stimulates macrophages via PI3K signaling}

Signaling to NF- $\kappa \mathrm{B}$ occurs via phospho-relay, and passes through several mitogen-activated protein kinase (MAPK) signaling nodes along the way, including phosphatidylinositide 3-kinase (PI3K; found upstream of the Akt pathway), c-Jun $\mathrm{NH}_{2}$-terminal kinase 1 (JNK), extracellular signal-regulated kinases 1 and 2 (ERK $1 \& 2$ ) and p38. To determine the mechanism of streptazolin-induced activation of $\mathrm{NF}-\kappa \mathrm{B}$, we targeted these four MAPKs using well-documented commercially available kinase inhibitors. By pretreating differentiated
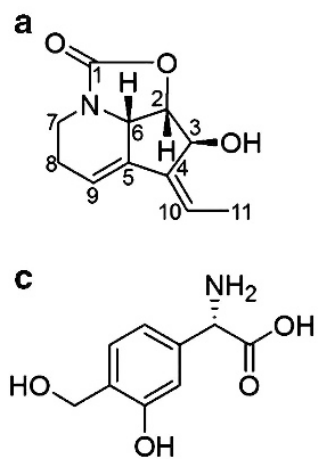

b

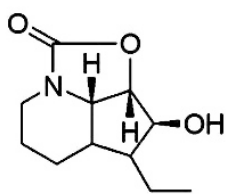

d

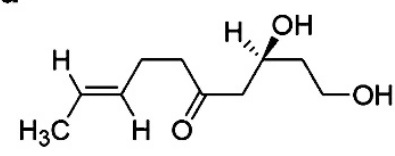

Figure 2 Structure of streptazolin and other natural products with macrophage-stimulating ability. Structure of streptazolin (a). Tetrahydrostreptazolin (b) was prepared by reducing the natural product, but was inactive in our nuclear factor $\mathrm{\kappa B}(\mathrm{NF}-\mathrm{kB})$ reporter assay. Forphenicol/ forphenicinol (c) and IC201 (d) are natural products with reported macrophage-stimulating activity, but importantly, are structurally dissimilar to streptazolin. 
Table 1 Complete NMR assignment of streptazolin (see also Figure 2a)

\begin{tabular}{|c|c|c|c|c|c|}
\hline$\#$ & 1H, p.p.m. & Multiplicity, J, Hz & 13C, p.p.m. & $\cos Y$ & $H M B C$ \\
\hline 1 & - & - & 158.6 & - & - \\
\hline 2 & 4.61 & $1 \mathrm{H}, \mathrm{d}, \mathrm{J}_{1}=6.8$ & 81.8 & 4.32 & $138.0 ; 143.3$ \\
\hline $3-\mathrm{OH}$ & 5.5 & $1 \mathrm{H}, \mathrm{d}, \mathrm{J}_{1}=6.0$ & - & 4.58 & $73.5 ; 81.8 ; 138.0$ \\
\hline 4 & - & - & 138.0 & - & - \\
\hline 5 & - & - & 143.3 & - & - \\
\hline $7 b$ & $3.26-3.30$ & $1 \mathrm{H}, \mathrm{m}$ & & & \\
\hline $8 a$ & $2.12-2.22$ & $1 \mathrm{H}, \mathrm{m}$ & 22.1 & $3.21,6.06$ & $39.5 ; 117.8 ; 143.3$ \\
\hline $8 b$ & $2.36-2.42$ & $1 \mathrm{H}, \mathrm{m}$ & & & \\
\hline 9 & 6.06 & $1 \mathrm{H}, \mathrm{m}$ & 117.8 & 2.4 & 73.5 \\
\hline 10 & 6.09 & $1 \mathrm{H}, \mathrm{q}, \mathrm{J}_{1}=7.1, \mathrm{~J}_{2}=7.6, \mathrm{~J}_{3}=7.1$ & 122.3 & 1.81 & $73.5 ; 143.3$ \\
\hline 11 & 1.81 & $3 \mathrm{H}, \mathrm{d}, \mathrm{J}_{1}=7.1$ & 14.4 & 6.09 & $73.5 ; 117.8 ; 122.3 ; 138.0 ; 143.3$ \\
\hline
\end{tabular}

Abbreviation: $\mathrm{HMBC}$, heteronuclear multiple bond correlation.
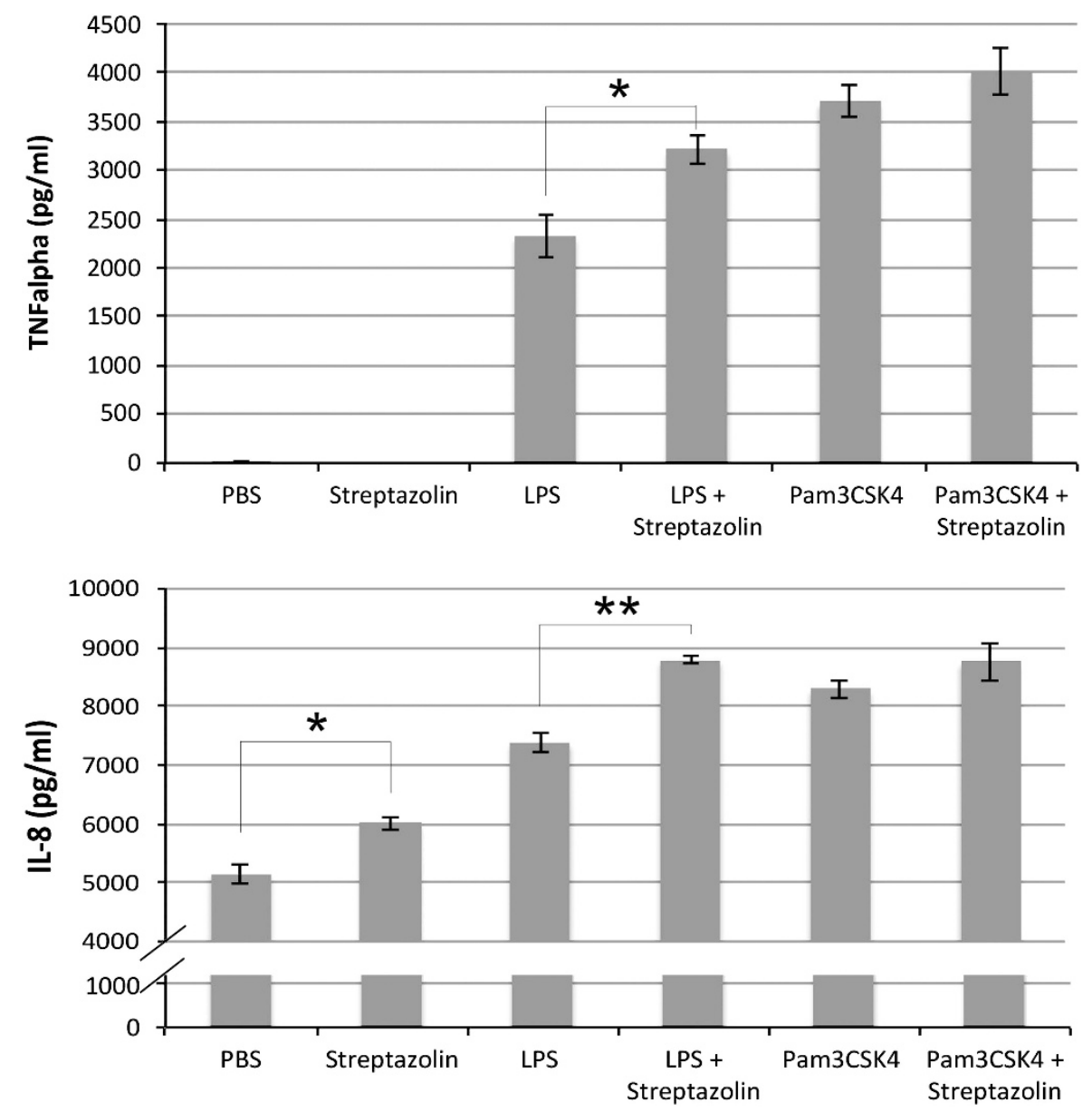

Figure 3 Cytokine release is triggered by exposure to streptazolin and Toll-like receptor (TLR) agonists. THP-1 macrophages were treated with streptazolin $\left(8 \mu \mathrm{g} \mathrm{ml}^{-1}\right)$ alone or in combination with the TLR agonists lipopolysaccharide (LPS; $10 \mathrm{ng} \mathrm{ml}^{-1}$ ) or Pam3CSK4 (500 ng ml ${ }^{-1}$ ), and cytokine induction was monitored by enzyme-linked immunosorbent assay (ELISA) at $24 \mathrm{~h}$. Although tumor necrosis factor- $\alpha$ (TNF $\alpha$ ) was not induced by streptazolin on its own, the response to LPS was significantly augmented in the presence of streptazolin. Interleukin-8 (IL-8) levels were significantly induced in the presence of streptazolin on its own and, as with TNF $\alpha$, boosted when streptazolin and LPS were added together compared with LPS alone. No significant increase in either cytokine was noted when streptazolin was added in combination with the TLR2 agonist Pam3CSK4. Results are an average of three experiments; “*' indicates a $P$-value of $<0.05$, ‘**' indicates a $P$-value of 0.001 . 


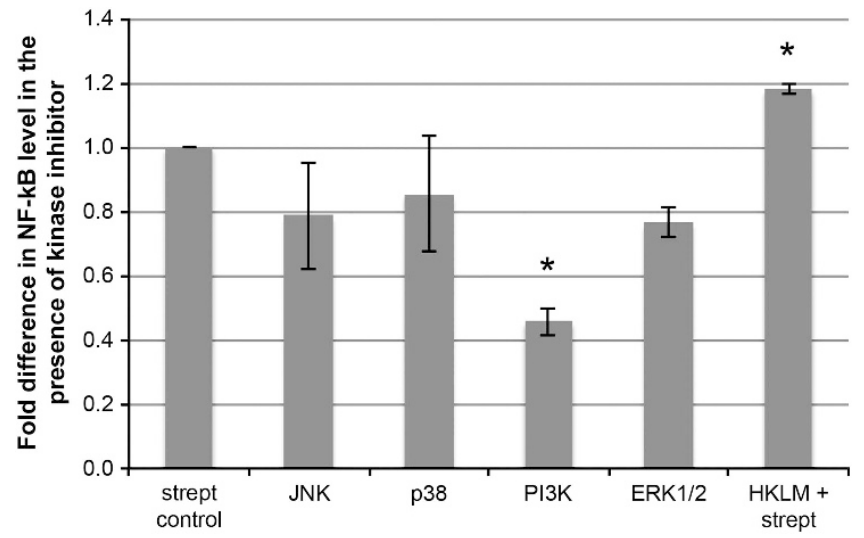

Figure 4 Streptazolin acts via phosphatidylinositide 3-kinase (PI3K) signaling. Cells were pretreated with inhibitors of four major signal transduction pathways for $1 \mathrm{~h}$ before treatment with $60 \mu \mathrm{g} \mathrm{ml}-1$ streptazolin, and then incubated overnight in the presence of both inhibitor and streptazolin. Streptazolin-induced nuclear factor $\mathrm{\kappa B}$ (NF-kB) activity was assessed at $24 \mathrm{~h}$, and values for the streptazolin alone control were set at 1 . Results for NF- $\mathrm{\kappa B}$ activity in the presence of streptazolin and inhibitors are expressed as a fold-difference vs the streptazolin alone control. Addition of the PI3K inhibitor LY294002 significantly reduced the level of NF-KB induction, relative to streptazolin alone, suggesting that streptazolin acts via PI3K-mediated signaling. Moreover, adding equal volumes of streptazolin and heat-killed Listeria monocytogenes (HKLM) resulted in additive levels of NF-kB activity, suggesting these ligands act through different receptors/ pathways. Results shown are the average of six experiments with s.d. shown, and are expressed as a fold difference in NF- $\mathrm{BB}$ activity in the presence of streptazolin and inhibitor compared with streptazolin treatment alone. Results indicated with $a$ '*' are highly significant, with $P$-values of $<0.005$.

THP-1 Blue cells with kinase inhibitors before treatment with streptazolin, we determined that streptazolin likely activates NF- $\kappa B$ via the PI3K-dependent pathway (Figure 4). Interestingly, adding streptazolin and HKLM in equal volumes resulted in an additive level of induction of NF- $\mathrm{KB}$ (Figure 4), suggesting that these molecules act through different receptors. We also monitored the supernatants of streptazolin-treated cultures of THP-1 Blue cells for SEAP activity over time to determine the time scale associated with streptazolininduced NF- $\kappa B$ activation. Over the course of $24 \mathrm{~h}$, streptazolin induced NF- $\kappa B$ on a time scale comparable to HKLM-positive controls (Figure 5a), suggesting that this molecule acts directly on macrophages to trigger reporter expression. Finally, we assessed the number of bacteria associated with macrophages (both bound and internalized) at early time points of infection (Figure 5b). Streptazolin treatment appears to increase the ability of macrophages to bind bacteria at early time points, which may contribute to increased bacterial killing in the presence of streptazolin.

\section{DISCUSSION}

The need for new strategies in the treatment of antibiotic-resistant bacterial infections is urgent and ever increasing. Stimulating the body's innate immune system is one such strategy, and natural products may represent an important source of immunostimulatory molecules. However, few reports exist in which this significant resource has been explored for macrophage-stimulating potential. ${ }^{7,10-13}$ Macrophages are an attractive target for immunostimulation because of both their phagocytic ability and their ability to elaborate cytokines and chemokines to recruit other immune cells. Furthermore, they present antigen to $\mathrm{CD} 4+\mathrm{T}$ cells of the adaptive immune system via major histocompatibility complex class II antigen, and therefore serve as a crucial bridging point between the innate and adaptive immune responses. We have developed a screening protocol based on the master transcriptional regulator $\mathrm{NF}-\kappa \mathrm{B}$ that is amenable to high-throughput screening, and may make identification of this type of molecule easier in the future. Our results indicate that natural products are a good source of NF- $\kappa \mathrm{B}-$ stimulatory molecules that have broad potential application: modulation of the innate immune system via NF- $\mathrm{BB}$ is essential not only for the elimination of microbial pathogens, but also for applications to cancer therapy. ${ }^{14}$

Our screen identified a strain of Streptomyces that produced a compound able to induce the NF- $\mathrm{B}$ pathway to a high degree, increase macrophage-mediated killing of $S$. mutans, and induced the elaboration of the important cytokines TNF $\alpha$ and IL- 8 in the presence of the TLR agonist LPS. We purified the small-molecule streptazolin from the culture supernatant by following NF- $\mathrm{B}$ activity, and identified it by HR-MS/MS, 1-D and 2-D NMR experiments. Streptazolin was first isolated over 30 years ago from a culture of Streptomyces viridochromogenes (strain Tü 1678), ${ }^{8}$ and has been subsequently isolated from Streptomyces luteogriseus (strain FH-S 1307) and Streptomyces sp. (strain FH-S 2184). ${ }^{15,16}$ It has an unusual ring system (hexahydro- $1 \mathrm{H}$-1-pyridine) composed of an internal urethane unit and an exocyclic ethylidene side chain. ${ }^{17}$ Urethane moieties are rare, but have been reported in the macrolactone irumamycin ${ }^{18}$ and the aglycon of the enediyne-type antibiotics calicheamicin and esperamicin. ${ }^{19,20}$ Although streptazolin itself has no antibiotic activity in our hands and only limited antimicrobial activities reported, ${ }^{16}$ structural variations have led to potent antimicrobial and cytotoxic derivatives. ${ }^{17}$ Streptazones $\mathrm{A}, \mathrm{B}_{1}$, $\mathrm{B}_{2}, \mathrm{C}$ and $\mathrm{D}$ are piperidine alkaloids that have been suggested to be intermediates in the synthesis of streptazolin. ${ }^{21}$ Several studies have reported testing streptazolin and/or its precursors against established tumor cell lines, with no reported cytotoxicity. ${ }^{13,22}$ The exception was streptazone A that was reported to be cytotoxic against selected human tumor cell lines. ${ }^{21}$ Our work and the work of others suggest that the activity of streptazolin resides in the reactivity of the conjugated diene system, such that reduction of this moiety eliminated immunostimulatory activity. Interestingly, the streptazolin precursor IC201 ((3S, 8E)-1,3-dihydroxy-8-decen-5one) has been reported to have immunomostimulatory activities in vivo, ${ }^{13}$ although it has no structural similarity to streptazolin (Figure 2d).

MAPKs are evolutionarily conserved serine/threonine protein kinases that are activated by external signals ${ }^{6}$ to trigger downstream signaling pathways involved in the regulation of growth, activation, differentiation and apoptosis of different cell types. ${ }^{23}$ We targeted four of the major macrophage MAPK signaling nodes leading to NF- $\kappa B$, and determined that streptazolin acts via PI3K. PI3K catalyzes the production of the second messenger phosphatidylinositol 3,4,5triphosphate that recruits and activates multiple downstream kinases including the Akt family of kinases. $^{24}$ The PI3K/Akt signaling pathway has been implicated in macrophage cell survival, proliferation, differentiation, intermediary metabolism and activation. Importantly, phosphatidylinositol 3,4,5-triphosphate activates the small guanosine-triphophatase (GTPase) Rac, resulting in the remodeling of the actin cytoskeleton that leads to phagocytosis. Phagocytosis and production of bactericidal reactive oxygen species are closely linked processes, ${ }^{25}$ and therefore stimulation of this pathway via streptazolin likely led to the increased rate of killing of S. mutans by treated macrophages. 
a

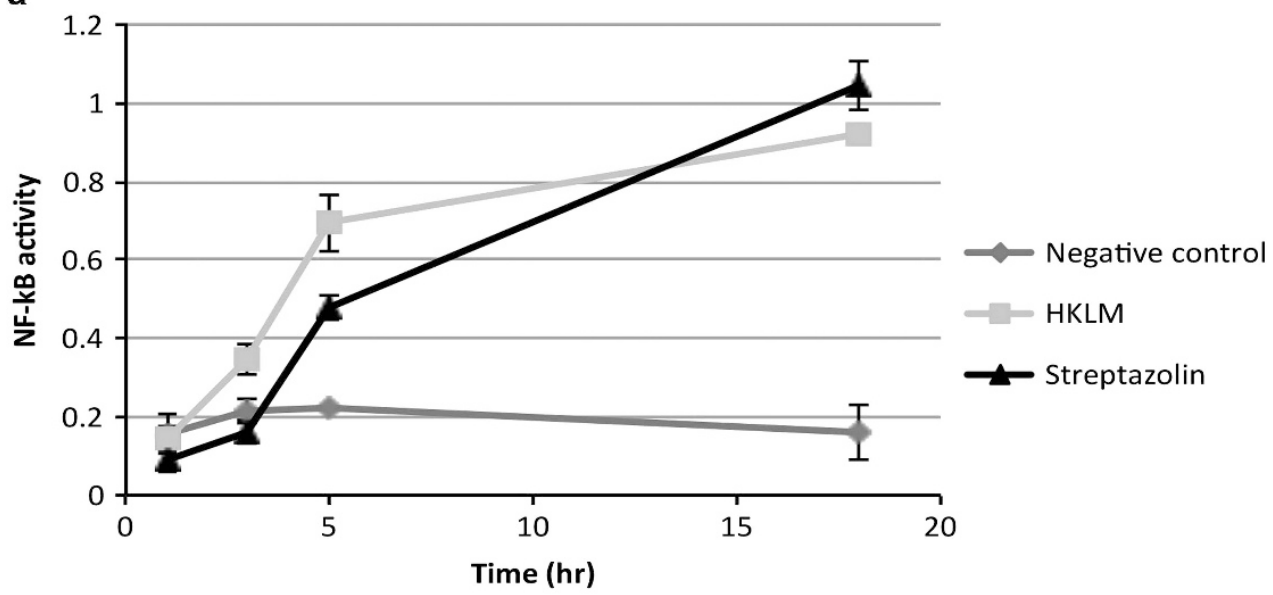

b

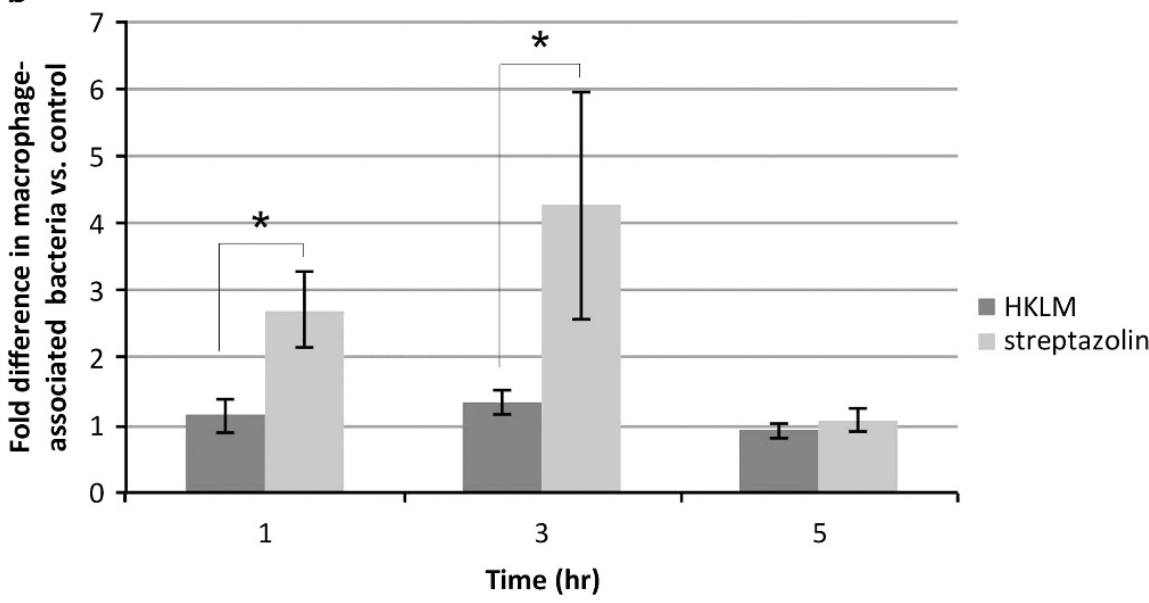

Figure 5 Streptazolin stimulates nuclear factor $\kappa B(N F-\kappa B)$ activity directly. In time course experiments, $60 \mu g \mathrm{ml}^{-1}$ streptazolin stimulated NF- $\mathrm{kB}$ activity on a timescale comparable to heat-killed Listeria monocytogenes (HKLM)-positive controls (a), suggesting that this molecule acts directly on macrophages to trigger reporter expression. Moreover, we assessed the number of bacteria associated with macrophages (both bound and internalized) at early time points of infection alone or in the presence of streptazolin or HKLM (b). Streptazolin treatment increased the number of Streptococcus mutans cells associated with macrophages at early time points, which may contribute to increased bacterial killing in the presence of streptazolin. Results are the average of three independent experiments with s.d. shown. Results indicated with a '*' indicate a $P$-value of $<0.05$.

\section{METHODS}

\section{Strains and growth conditions}

THP1-Blue NF- $\mathrm{B}$ cells (Invivogen) were maintained in RPMI-1640 supplemented with $10 \%$ fetal bovine serum (Invitrogen, Grand Island, NY, USA) at $37^{\circ} \mathrm{C}$ in a humidified chamber under $5 \% \mathrm{CO}_{2}$. THP1-Blue cells were differentiated by addition of $100 \mathrm{~nm}$ phorbol 12-myristate 13-acetate (PMA) (Sigma, St Louis, MO, USA) to culture media, and incubated for $72 \mathrm{~h}$. S. mutans UA159 was grown in Todd Hewitt-Yeast Extract medium (18 g ToddHewitt Broth, $3 \mathrm{~g}$ yeast extract in $11 \mathrm{dH}_{2} \mathrm{O}$ ) in a stationary incubator at $37^{\circ} \mathrm{C}$ in an atmosphere supplemented with $5 \% \mathrm{CO}_{2}$.

\section{Preparation of the NPL}

A heterogeneous collection of Actinomycetes from geographically disparate locations was assembled in-house for screening purposes. To generate the Natural Product Library (NPL) for screening, monocultures were seeded from frozen spore stocks into SAM medium and grown for 6 days at $30^{\circ} \mathrm{C}$ with agitation. These seed cultures were used to streak Bennett's agar for confluent growth, and grown at $30^{\circ} \mathrm{C}$ in a stationary incubator for a further 6 days. Agar and cells were then extracted with methanol. Extracts were dried and resuspended in DMSO for screening.

\section{Screening}

THP1-Blue monocytes were seeded at $\sim 3 \times 10^{4}$ cells per well in 96-well plates in the presence of PMA, and allowed to differentiate for $72 \mathrm{~h}$. A total of 560 NPL extracts were added at $0.5 \% \mathrm{vol} / \mathrm{vol}$ to existing culture media following differentiation, and cells were returned to the incubator for a further $18 \mathrm{~h}$. HKLM (Invivogen) was used as a positive control for THP1-Blue activation, and DMSO was used as a vehicle control (both also at $0.5 \% \mathrm{vol} / \mathrm{vol}$ ). After $18 \mathrm{~h}$, culture supernatants were sampled aseptically and assessed for NF- $\mathrm{KB}$ activity using the SEAP detection reagent Quanti-Blue (Invivogen) according to the manufacturer's directions. Macrophages expressing NF- $\mathrm{KB}$ at levels equal to or exceeding the levels induced by HKLM were then probed for killing ability using the model Gram-positive organism S. mutans.

\section{Killing assay}

Macrophages were treated for $18 \mathrm{~h}$ with either HKLM or NPL extract and assessed for NF- $\kappa \mathrm{B}$ activity before proceeding with killing assays. In activated macrophages, spent media were completely removed and replaced with RPMI1640 supplemented with fetal bovine serum and S. mutans at an approximate multiplicity of infection of 100 in minimal volume. Macrophages were returned to the incubator for $2 \mathrm{~h}$ to allow for bacterial binding, uptake and 
killing. Culture supernatants were then diluted with an equal volume of media containing $50 \mu \mathrm{g} \mathrm{ml}^{-1}$ gentamicin and $40 \mathrm{U} \mathrm{ml}^{-1}$ penicillin/streptomycin and incubated for $1 \mathrm{~h}$ at $37^{\circ} \mathrm{C}$ to kill any extracellular bacteria. An aliquot of the antibiotic-treated supernatant was plated out to ensure sterilization, and media were removed and replaced with $\mathrm{dH}_{2} \mathrm{O}$ to lyse macrophage and liberate surviving intracellular bacteria. Intracellular bacteria were serially diluted and plated to THYE agar for enumeration.

\section{Antibiotic activity vs Streptococcus}

To ensure the NPL extracts chosen for follow-up did not have spurious antibiotic activity, supernatants were incubated with $S$. mutans overnight in liquid culture at $37^{\circ} \mathrm{C}$. Growth was assessed by OD at $600 \mathrm{~nm}$. Purified streptazolin was also assessed for antibiotic activity via liquid dilution MIC as per standard protocols up to maximum concentration tested of $512 \mu \mathrm{g} \mathrm{ml}^{-1}$.

\section{Activity-guided natural product purification}

Three strains of Streptomyces selected as 'top hits' (that is, inducing high NF$\kappa \mathrm{B}$ activity and increasing killing of $S$. mutans compared with HKLM controls) were used to validate screening results, and were grown on solid Bennett's agar from frozen stock for 6 days before inoculating a liquid culture. The highest activity upon regrowth was obtained from strain WAC1325 which was then grown on a larger scale for activity-guided purification. For large-scale production, colonies from solid-phase cultures were inoculated into either Bennett's media or Maltose-Yeast-Glucose medium (10 g maltose, $4 \mathrm{~g}$ yeast extract, $4 \mathrm{~g}$ glucose, $11 \mathrm{H}_{2} \mathrm{O}$ at $\mathrm{pH} 7.5$ ) in baffled flasks, and grow at $30^{\circ} \mathrm{C}$ with agitation for 6 days. Production of macrophage-stimulating compound occurred most abundantly in MYG medium in a medium volume of $250 \mathrm{ml}$ in a 11 flask. Four such flasks were grown for 6 days and cells were then collected by centrifugation. Supernatants were mixed with Celite resin $(16 \mathrm{~g}$ Celite/11 culture) and incubated at room temperature for $1 \mathrm{~h}$ with agitation. The resin was removed by filtration, and both spent medium and pellet were extracted with ethyl acetate. Extracts were concentrated under vaccuum, and fractionalized on a CombiFlash system using a silica resin and a stepwise gradient of chloroform $/ 10 \%$ methanol in chloroform. Active compound eluted at $5 \%$ methanol. Activity of the extract was assessed following each purification step using both NF-KB activity assays and killing assays.

\section{Kinase inhibitor assays}

Kinase inhibitors SB203580 (targeting p38, $10 \mu \mathrm{M}$ ), SP600125 (c-Jun $\mathrm{NH}_{2}$ terminal kinase 1, $25 \mu \mathrm{M}$ ), LY294002 (PI3K, $10 \mu \mathrm{M}$ ) and U0126 (extracellular signal-regulated kinases 1 and 2, $25 \mu \mathrm{M}$ ) were added to differentiated THP-1 Blue macrophages in complete media and incubated for $1 \mathrm{~h}$ at $37^{\circ} \mathrm{C}$ before the addition of streptazolin. Cells were incubated overnight in the presence of both inhibitor and streptazolin, and NF- $\kappa \mathrm{B}$ activity was assessed using the SEAP reporter assay at $24 \mathrm{~h}$ as described.

\section{Analytical LC-MS/NMR analysis}

LC-ESI-MS data were obtained by using an Agilent 1100 Series LC system (Agilent Technologies, Santa Clara, CA, USA) and a QTRAP LC/MS/MS System (Applied Biosystems, Grand Island, NY, USA). The reverse-phase HPLC was performed using $\mathrm{C}_{8}$ column (Zorbax $5 \mu \mathrm{m}, 4.6 \times 150 \mathrm{~mm}$ ) with Agilent 1100 LC binary pump at a flow rate of $1 \mathrm{ml} \mathrm{min}^{-1}$ under the following conditions: isocratic $5 \%$ solvent B (0.05\% formic acid in acetonitrile) and $95 \%$ solvent A $(0.05 \%$ formic acid in water) for $1 \mathrm{~min}$, followed by a linear gradient to $97 \%$ B over $6 \mathrm{~min}$.

Compound structure was confirmed by 1-D and 2-D NMR experiments on a Bruker AVIII $700 \mathrm{MHz}$ instrument (Billerica, MA, USA) equipped with a cryoprobe in deuterated DMSO solvent. Chemical shifts are reported in p.p.m. relative to tetramethyl silane using the residual solvent signal as an internal signal. HR-MS were obtained using Thermo Fisher-XL-Orbitrap Hybrid mass spectrometer (Thermo-Fisher, Bremen, Germany) equipped with electrospray interface operated in positive ion mode.

\section{Enzyme-linked immunosorbent assay cytokine assays}

THP-1 Blue cells were seeded and differentiated as described, and treated with LPS at $10 \mathrm{ng} \mathrm{ml}^{-1}$, the synthetic TLR2 ligand Pam3CSK4 at $500 \mathrm{ng} \mathrm{ml}^{-1}$ and purified streptazolin at $8 \mu \mathrm{g} \mathrm{ml}^{-1}$ alone and in combination for $24 \mathrm{~h}$. Secreted cytokines were measured using the MILLIPLEX Map Human Th17 Magnetic Bead Panel and a MAGPIX multiplexing instrument (Millipore, Billerica, MA, USA) according to the manufacturer's recommendations.

\section{CONCLUSION}

In summary, we have isolated the macrophage-stimulating compound streptazolin from a screen of microbial natural products. Structure/ activity relationship studies indicate that the conjugated diene moiety is responsible for NF- $\kappa \mathrm{B}$ activation, which occurs via the PI3Kdependent pathway. Macrophages stimulated with streptazolin are better able to kill $S$. mutans in vitro, and elaborate immunostimulatory cytokines like TNF $\alpha$ and IL- 8 in the presence of bacterial LPS. Searching for alternative ways to treat bacterial infections is becoming increasingly important in an age where antibiotic resistance is rampant. Immunostimulatory strategies represent a promising alternative to antibiotics, and natural products represent an underexplored resource for this type of molecule that may be mined in the future.

\section{CONFLICT OF INTEREST}

The authors declare no conflict of interest.

\section{ACKNOWLEDGEMENTS}

This work was partly supported by a Natural Sciences and Engineering Research Council Grant (237480), a Canada Research Chair in Biochemistry (to GDW), a Canadian Institutes for Health Research (CIHR) Post-doctoral Fellowship (to JAP), the McMaster Immunology Research Centre and the M.G. DeGroote Institute for Infectious Disease Research (to DMEB) and a fellowship from the Canadian Thoracic Society (to CPV).

1 Beutler, B. Innate immunity: an overview. Mol. Immunol. 40, 845-859 (2004).

2 Verschoor, C. P., Puchta, A. \& Bowdish, D. M. The macrophage. Met. Mol. Biol. 844 139-156 (2012)

3 Beinke, S. \& Ley, S. C. Functions of NF-kappaB1 and NF-kappaB2 in immune cell biology. Biochem. J. 382(Pt 2), 393-409 (2004).

4 Vanek, Z. M. J. \& Curdova, E. Immunomodulators isolated from microorganisms. Folia Microbiol. 36, 99-111 (1991).

5 Hancock, R. E., Nijnik, A. \& Philpott, D. J. Modulating immunity as a therapy for bacterial infections. Nat. Rev. Microbiol. 10, 243-254 (2012).

6 Kakeya, H. et al. Isolation and biological activity of a novel cytokine modulator, cytoxazone. J. Antibiot. (Tokyo) 51, 1126-1128 (1998)

7 Lkhagvaa, B et al. Bestatin, an inhibitor for aminopeptidases, modulates the production of cytokines and chemokines by activated monocytes and macrophages. Cytokine 44, 386-391 (2008).

8 Drautz, H., Zähner, H., Kupfer, E. \& Keller-Schierlein, W. Isolierung und Struktur von Streptazolin. Helv. Chimica. Acta 64, 1752-1765 (1981).

9 Kozikowski, A. P. \& Park, P. U. Total synthesis of streptazolin: an application of the aza analogue of the ferrier rearrangement. J. Am. Chem. Soc. 107, 1763-1765 (1985).

10 Watanabe, Y., Tawara, S., Mine, Y. \& Kikuchi, H. Immunoactive peptides, FK 156 and FK 565. IV. Activation of mouse macrophages. J. Antibiot. (Tokyo) 38, 1781-1787 (1985).

11 Ohhara, N., Okamura, S., Otsuka, T., Harada, M. \& Niho, Y. Stimulatory effect of forphenicinol on normal human bone marrow granulocyte-macrophage progenitor cells mediated by T-lymphocytes. Biomed. Pharmacother. 41, 425-428 (1987).

12 Hamada, M., Sonotake, E., Yamamoto, S. \& Moriguchi, S. Conagenin derived from Streptomyces roseosporus enhances macrophage functions. J. Antibiot. (Tokyo) 52, 548-551 (1999).

13 Mizutani, S. et al. Biological activities of IC201 ((3S,8E)-1,3-dihydroxy-8-decen-5one), a low molecular weight immunomodulator produced by Streptomyces. J. Antibiot. (Tokyo) 42, 952-959 (1989).

14 De Palma, M. \& Lewis, CE. Macrophage regulation of tumor responses to anticancer therapies. Cancer Cell 23, 277-286 (2013).

15 Grabley, S. et al. Secondary metabolites by chemical screening. 4. Detection, isolation and biological activities of chiral synthons from Streptomyces. J. Antibiot. (Tokyo) 44, 797-800 (1991) 
16 Grabley, S. et al. Secondary metabolites by chemical screening. 21. Clonostachydiol, a novel anthelmintic macrodiolide from the fungus Clonostachys cylindrospora (strain FH-A 6607). J. Antibiot. (Tokyo) 46, 343-345 (1993).

17 Mayer, M. \& Thiericke, R. Biosynthesis of Streptazolin. J. Org. Chem. 58, 3486-3489 (1993).

18 Omura, S. et al. Irumamycin, a new antibiotic active against phytopathogenic fungi. J. Antibiot. (Tokyo) 35, 256-257 (1982).

19 Konishi, M. et al. Esperamicins, a novel class of potent antitumor antibiotics. I. Physico-chemical data and partial structure. J. Antibiot. (Tokyo) 38, 1605-1609 (1985).

20 Ikemoto, N. et al. Calicheamicin-DNA complexes: warhead alignment and saccharide recognition of the minor groove. Proc. Natl Acad. Sci. USA 92, $10506-$ 10510 (1995).
21 Puder, C., Krastel, P. \& Zeeck, A. Streptazones A, B(1), B(2), C, and D: new piperidine alkaloids from streptomycetes. J. Nat. Prod. 63, 1258-1260 (2000).

22 Puder, C., Loya, S., Hizi, A. \& Zeeck, A. New co-metabolites of the streptazolin pathway. J. Nat. Prod. 64, 42-45 (2001).

23 Comalada, M., Lloberas, J. \& Celada, A. MKP-1: a critical phosphatase in the biology of macrophages controlling the switch between proliferation and activation. Eur. J. Immunol. 42, 1938-1948 (2012).

24 Zhou, D. et al. Macrophage polarization and function with emphasis on the evolving roles of coordinated regulation of cellular signaling pathways. Cell. Signal. 26, 192-197 (2013).

25 Harada, T. et al. Phagocytic entry of Legionella pneumophila into macrophages through phosphatidylinositol 3,4,5-trisphosphate-independent pathway. Biol. Pharm. Bull. 35, 1460-1468 (2012) 